\title{
Primary cardiac tumours: how well can prenatal diagnosis "predict” postnatal outcome?
}

\author{
Habanova $\mathrm{H}^{1}$, Kaldararova $\mathrm{M}^{2}$, Tittel $\mathrm{P}^{2}$, Kovacikova $\mathrm{L}^{2}$, Cunderlik $\mathrm{A}^{1}$, Rusnak $\mathrm{I}^{1}$ \\ 1st Clinic of Gynecology and Obstetrics, Slovak Medical University and University Hospital Bratislava, \\ Slovakia. hana.habanova@gmail.com
}

\section{ABSTRACT}

OBJECTIVE: Primary foetal cardiac tumours are rare congenital malformations. They can cause a flow obstruction, arrhythmias and can lead to cardiac failure, hydrops or death. Postnatal management is based on patient's clinical and hemodynamic impairment.

METHODS: We retrospectively reviewed data from 2009-2019 from our gynaecology clinic and also data regarding postnatal follow-up from our partner paediatric institution.

RESULTS: In this period, we diagnosed six cases with foetal cardiac tumours. In four cases, multiple rhabdomyomas were present. Three patients did not have serious complications pre- or postnatally. In one case, tumours were obliterating both the inflow and the outflow of the left ventricle. The child died at three months of age. Tuberous sclerosis was confirmed in all the cases with rhabdomyomas. One child had a fibroma filling the left ventricle. Despite an uneventful prenatal period, the patient got postnatally symptomatic. Tumour was considered inoperable and the child died at the age of five months.

In one case a single right ventricular unspecified tumour was diagnosed, without any complications. CONCLUSION: Prognosis closely depends on early diagnosis, clinical manifestations and the possibility of surgical tumour removal if necessary. In confirmed rhabdomyomas, tests for tuberous sclerosis are mandatory (Tab. 1, Fig. 2, Ref. 18). Text in PDF www.elis.sk KEY WORDS: rhabdomyoma, fibroma, prenatal diagnosis, ultrasound, tuberous sclerosis.

\section{Introduction}

The primary foetal cardiac tumours are rare congenital malformations with an incidence of between $0.08-0.20 \%$ (1). Although the incidence of foetal cardiac tumours is very low, their importance lies in a heterogeneous clinical manifestation and a possible serious impact on the foetus as well as the newborns. For example, in recent years the search for an alternative therapeutic possibility promoted the introduction of a mammalian target of rapamycin (mTOR) inhibitors in the treatment of inoperable rhabdomyomas (2). The vast majority of primary foetal cardiac tumours are histologically benign since the malignant type of cardiac tumours are extremely rare in foetuses. The most common histological types are rhabdomyoma, fibroma, teratoma, haemangioma and myxoma (3).

The most common type of foetal cardiac tumour is rhabdomyoma, which is found in $60 \%$ of diagnosed cases. Rhabdomyoma is a benign, usually multifocal (in $90 \%$ of cases), hyperechogenic tumour (3) that is usually localized in the right or left ventricle. The tumour may spontaneously regress after the birth.

${ }^{1} 1$ st Clinic of Gynecology and Obstetrics, Slovak Medical University and University Hospital Bratislava, Slovakia, and ${ }^{2}$ Children’s Cardiac Center, National Institute of Cardiovascular Diseases, Bratislava, Slovakia

Address for correspondence: H. Habanova, 1st Clinic of Gynecology and Obstetrics, Slovak Medical University and University Hospital Bratislava, Slovakia.

Phone: +421.915.185555
There is a well-known association between rhabdomyoma and tuberous sclerosis with an incidence of up to $50 \%$ of the cases (4). Tuberous sclerosis is an autosomal dominant disorder with a variable expressivity, and most of the cases are caused by de novo mutation (5).

The possible clinical manifestations of rhabdomyoma (prenatally but similarly also postnatally) are ventricular inflow or outflow tract obstruction and/or arrhythmias, leading to the foetal hydrops and cardiogenic shock. The prognosis of children and foetuses with a rhabdomyoma depends on its clinical manifestation: from follow-up, administering of antiarrhythmic drugs to surgical removal of the obstructing tumour (3). In the recent years, in the cases of inoperable multifocal rhabdomyomas, everolimus or sirolimus (mTOR inhibitors) are used for tumour shrinkage $(6,7)$. These therapeutic agents are nowadays being used in many fields of medicine, e.g. in treatment of atherosclerosis (8).

The second most common foetal cardiac tumour is fibroma. Fibromas are histologically benign, usually solitary tumours of fibroblast origin, with a hyperechogenic appearance during echocardiographic examination. They may be pedunculated, located usually in left ventricle, and, compared to rhabdomyomas, they never regress in size postnatally (3). Their most common clinical manifestations are arrhythmias and valvular obstruction. The prognosis of children with fibromas depends on the size of the tumour; in cases with an inoperable tumour (where resection is difficult), heart transplantation may be indicated (9). 


\section{5-319}

Teratoma is a very rare type of primary foetal cardiac tumour. In most cases it is a solitary, intrapericardially localized, cystic tumour with mixed echogenicity. Pathognomic for teratoma is the presence of pericardial effusion. The clinical manifestation of the tumorous mass may include foetal hydrops (as a result of compromised venous return to the right heart due to a growing tumour) or cardiac tamponade (as result of a growing mass or large amount of pericardial effusion) (10).

The last mentioned type of foetal cardiac tumour is myxoma, which is very rare during the prenatal period and in newborns. However, later in childhood myxomas are one of the most common cardiac tumours (11). They are usually ovoid, pedunculated tumours with a clinical manifestation of embolism and intracardiac obstruction (12).

The prenatal diagnosis of cardiac tumours is done by ultrasound. During the examination, the localization, number, size and echogenicity are described. In addition to reviewing the detailed morphology of the heart, an evaluation of the heart rate and hemodynamic by using Doppler spectra is mandatory. A suspicion of this diagnosis is in most of the cases made during a routine obstetric ultrasound (the discovery of a mass in the foetal heart, foetal arrhythmia, or pericardial effusion). Although foetal cardiac tumours can already be diagnosed in the second trimester, in some cases, the diagnosis is made in the third trimester (13). Also screening for associated foetal anomalies is necessary (14).

\section{Materials and methods}

In our clinic, we retrospectively analysed all the cases with the prenatal diagnosis of primary foetal cardiac tumours in the period from 2009-2019.

In all the cases, the pregnant women were referred either to our clinic or the Children's Cardiac Center, because of the identification of an abnormal cardiac mass during a routine obstetric ultrasound examination. Once the suspicion of foetal cardiac tumour was made, the pregnant woman was referred to our clinic or to our collaborating institution, the Children's Cardiac Center. After the confirmation of the diagnosis, further follow-up of the pregnancy consisted of regular controls in our gynaecology department and foetal echocardiographic examinations in the Children's Cardiac Center. Together we planed the optimal management of each case with regard to time and a mode of delivery. In our country, our clinic serves as the centre for prenatal care and delivery for pregnant women with prenatally diagnosed foetal cardiac defects (comprising foetal cardiac tumours).

During the analysis, we gathered information regarding the mother and the pregnancy, as well as the foetus: the age of mother and the week of gestation at referral, the gestation age at birth and the mode of delivery. From the echocardiographic examination: the localization, size, number and appearance of the tumours were documented. We also looked for the signs of foetal cardiac compromise and the presence of other congenital malformations and cardiac defects. In addition, postnatal data were documented: the infant's sex, birth weight and Apgar score. We also obtained postnatal follow-up data on the management of the patients from the Children's Cardiac Centre. The data included information on postnatal changes in the size of the tumours, the presence of tuberous sclerosis (in patients with diagnosed rhabdomyomas), the operational interventions needed, the presence of arrhythmias, the further clinical course, and cardiac complications.

Note: one of the cases was used by way of demonstration as a part of an article in another language (Slovak) (15).

\section{Results}

In the selected period of years, we had six cases with prenatally diagnosed foetal cardiac tumours.

\section{Maternal characteristics}

Among pregnant women with prenatally diagnosed primary foetal cardiac tumours, the average age was 32.8 years (23-39 years) at the time of referral. Most of the cases were diagnosed in the third trimester (from 23 to 38 weeks of gestation). The average week of gestation in the studied cases at the time of birth was the 40th week (38-41 weeks of gestation).

\section{Prenatal findings}

In our foetal series, we found four patients with rhabdomyomas (66.7\%), one patient with a fibroma (16.7\%), and one patient had an unidentified cardiac tumour (16.7\%).

In three cases, a typical picture of rhabdomyomas was foundwith multiple hyperechogenic tumours of different size, located both in the right and the left ventricles (Fig. 1).

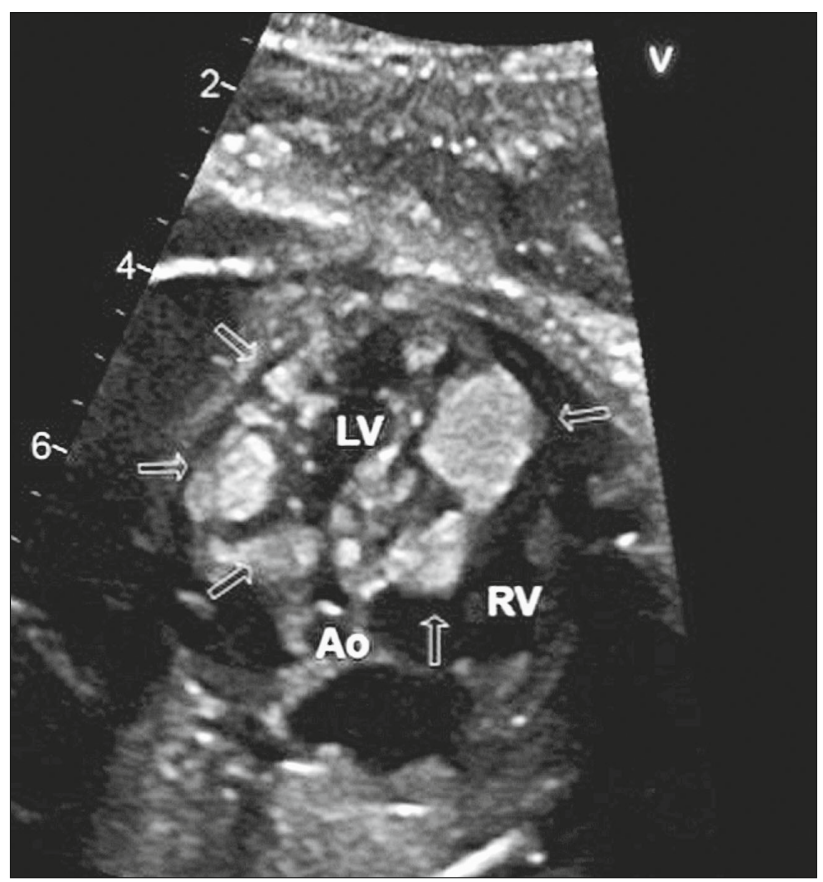

Fig. 1. Multiple rhabdomyomas located in both ventricles and interventricular septum. Ao - aorta, LV - left ventricle, RV - right ventricle. 


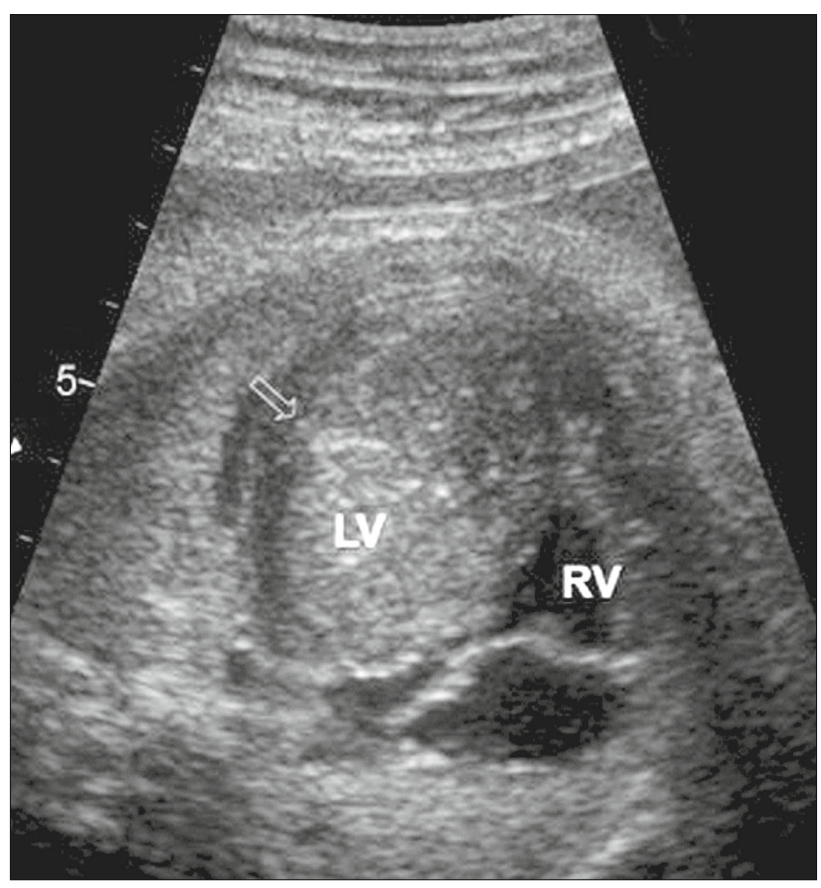

Fig. 2. Fibroma obliterating the left ventricle. $L V$ - left ventricle, RV - right ventricle.

In one case, an atypical picture of rhabdomyomas was present, where the tumours were obliterating both the inflow (mitral valve) and the outflow of the left ventricle.

In one foetus, a large hyperechogenic fibroma, almost completely filling the left ventricle was diagnosed (Fig. 2).

In one prenatally diagnosed mass, the tumour type was not specified according to ultrasound findings. It was localized in the right ventricle without any progression in growth during the prenatal period.

In one case of typical multiple rhabdomyomas, amniocentesis was performed, and the diagnosis of tuberous sclerosis was confirmed. In all foetuses, prenatally no other structural malformations or cardiac defects were diagnosed.

During our prenatal follow-up, we didn't find any signs of cardiac decompensation or arrhythmias in any of the cases. All foetuses had normal biometric parameters, amniotic fluid volume and Doppler blood flow in the umbilical artery on prenatal ultrasound scans.

\section{Mode of delivery and the characteristics of newborns}

In our series, four pregnant women delivered vaginally, in two cases a Caesarean section from an obstetric indication was performed. In four cases, the infant's sex was male, in two female. The average birth weight was 3440 grams (2930-4070 grams), and the immediate postnatal adaptation, according to Apgar score, was 8.6 in the 1 st and 9.4 in 5th (5-10 in 1st minute and 7-10 in 5th minute).

\section{Postnatal follow-up}

Cases 1-3. Three patients had typical multiple rhabdomyomas located in both the right and the left ventricles. None of these were causing flow obstruction, and none of the patients needed a surgical intervention postnatally. In two cases, the tumours regressed in size during follow-up, and in one case, the tumours remained constant. After birth, in two of these patients benign transient arrhythmias (extrasystoles) were observed. The diagnosis of tuberous sclerosis was confirmed in all the three patients (in one already prenatally).

Case 4. This patient had an atypical location of the tumours: two large rhabdomyomas were diagnosed in the left atrium (the mitral valve), as well as in the left ventricle and a further smaller one in the right ventricle. After birth, the child had signs of severe mitral valve obstruction with almost no antegrade flow to the left ventricle with a complex single-ventricle hemodynamic. Soon postnatally also arrhythmias developed. Palliative surgical bilateral pulmonary artery banding was performed, and Prostaglandin infusion was continued to keep the arterial duct open. It was considered not possible to resect the tumours. Sirolimus was therefore used to promote the shrinkage of the tumours. After 3 weeks of therapy, significant antegrade flow in left ventricle was achieved. Nevertheless, with the regression of the tumours, moderate to severe mitral and aortic valve regurgitations appeared. Clinical signs of severe heart failure persisted, and the patient died at the age of three months. The patient had a confirmed diagnosis of tuberous sclerosis.

Case 5. In this case, a single tumorous mass, fibroma, almost completely obliterated

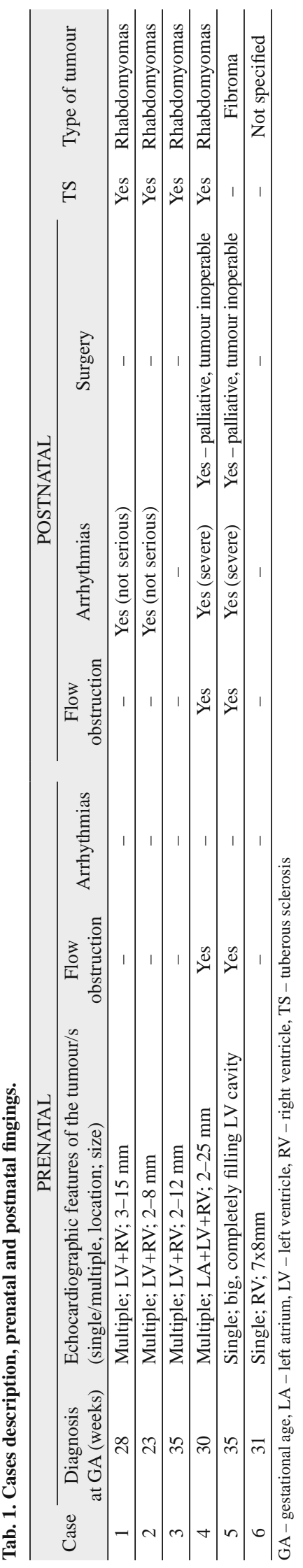


the cavity of the left ventricle. The patient had signs of severe left outflow tract obstruction after birth. The tumour was considered inoperable, and only palliative surgery (aortopexy) was performed. The child had severe arrhythmias and signs of heart failure, ultimately dying at the age of five months.

Case 6. The last patient from the study group had an unspecified single tumour of the right ventricle without signs of flow obstruction or any presence of arrhythmias. There was no change in the size of the tumour during the postnatal follow-up, and a surgical removal of the mass was not needed.

A summary of all the cases on both prenatal and postnatal findings is mentioned in Table 1.

\section{Discussion}

Primary foetal cardiac tumours are rare congenital malformations. According to their echocardiographic appearance, in most cases the tumour type can be specified. In addition to structural characteristics, the description should always define the number of tumours (single/multiple), their localization (in the left or right ventricle or atrium, proximity to any valve or the conduction system), and the hemodynamic impact on the circulation (flow obstruction or valve regurgitation).

\section{Tumour types}

The most common prenatal tumour type is rhabdomyoma, which was present in our research in $67 \%$ of the prenatally diagnosed cases. All these patients had multiple tumours localized in both the right and the left ventricles. The tumours differed in the size from a few millimetres to few centimetres. This finding corresponds with the usual appearance of rhabdomyomas on ultrasound (Fig. 1).

One of our patients with rhabdomyomas had also a very unusual location - a large tumour was present in the left atrium growing in the mitral valve orifice. This was a very unfavourable location not only causing hemodynamically significant flow obstruction to the left ventricle, but being also impossible to remove surgically postnatally without a mitral valve destruction.

In rhabdomyomas not only the size of the tumours, but the location itself is of great importance. There is evidence that they can impair the conduction system of the foetal heart and lead to arrhythmias (16).

Despite our fear, in our series the tumorous masses did not cause prenatally any foetal compromise (even in patients with flow obstruction), and no arrhythmias were prenatally detected.

Three of our patients with rhabdomyomas had a favourable postnatal follow-up without any need for intervention. This confirmed the typically described clinical course with a possible postnatal regression of tumours and a good overall long-term prognosis.

By contrast, we also described a patient with a severe flow obstruction due to the localization of the tumours. In this case, the hemodynamic and clinical impact of the tumours was so severe that has led to the patient's death even after Sirolimus administration and the regression of the size of the tumours. Despite the usually described $50 \%$ incidence of tuberous sclerosis, in our series, this was confirmed in all the patients with prenatally diagnosed rhabdomyomas. The prenatal finding of cardiac tumorous masses can perhaps also accelerate the early diagnosis of tuberous sclerosis, even before a neurological manifestation appears; this may be of immense clinical importance for the patient.

In our prenatal study, we had one patient with cardiac fibroma, with the corresponding incidence of $17 \%$. Fibromas are benign hyperechogenic tumours, which are usually located in the ventricles. This was also documented in our case, where a large fibroma filling the left ventricle was diagnosed (Fig. 2). Despite a severe flow obstruction, the tumour prenatally did not cause clinical disturbances or arrhythmias. However, postnatally the child required an early intensive care.

Unlike rhabdomyomas, fibromas do not regress in size after birth, and in the case of a large tumour with a clinical manifestation, surgical removal, if possible, is the treatment of choice for children. In our case, the tumour was inoperable, and the clinical course of the patient, due to hemodynamic impairment and arrhythmias, was very unfavourable, and the patient died at the age of five months.

In our 11-year prenatal series we did not have patients with a confirmed teratoma or myxoma.

In one case of prenatally diagnosed foetal cardiac tumour, the exact type of tumour was not specified. It was a single tumour located in right ventricle, without any change in size during the postnatal follow-up. Since it did not cause any problems and has not required a surgical removal, we do not have the histopathological confirmation of the tumour type. Sometimes the differential diagnosis of a single cardiac mass can be difficult, when we are depending only on ultrasound findings $(17,18)$.

\section{Prenatal risk stratification}

A very interesting fact was that none of our patients had signs of hemodynamic compromise prenatally. This could perhaps be explained by the specific foetal hemodynamic itself, which was able to compensate for even severe ventricular flow (inflow or outflow) obstruction. However, if severe valve regurgitation were to develop prenatally due to the presence of the tumour, this presumably would not be tolerated so well.

Despite the optimistic uneventful prenatal course, the postnatal physiological changes of the hemodynamic in patients with a flow obstruction led to clinical deterioration soon after birth, and they required an intensive paediatric cardiac care.

In patients with cardiac tumours, also the question of possible arrhythmias needs to be addressed carefully since arrhythmias can occur anytime prenatally or postnatally. They develop frequently as the result of clinical decompensation (heart failure) and represent prenatally a very unfavourable prognostic sign. Moreover, in some cases, also the critical localization of the tumour (even a small one) can cause a severe damage to the conduction system (16), and the foetus can develop life-threatening arrhythmias.

In our patients, we did not observe any arrhythmias prenatally since they were hemodynamically stable and luckily none of the tumours was located near the conduction system.

From the gynaecologist's point of view, it is very important not only to diagnose the foetuses with cardiac tumours, but also 
to stratify the high-risk patients. This is especially important in cases, where a significant flow obstruction or arrhythmias make it necessary to monitor them closely throughout the pregnancy to rule out possible prenatal complications. In these pregnancies, also the optimal labour planning (regarding the time and mode of delivery) with a paediatric cardiac centre is mandatory - since in the high risk patients we need to expect an early clinical worsening after delivery. However, patients without any hemodynamic impairment or arrhythmias may have a very favourable postnatal long-term clinical course.

\section{Prenatal diagnosis}

In addition to our six cases of foetal cardiac tumours during the selected period (2009-2019), there were another eight patients (newborns, infants and young children up to the age of 2 years) with primary cardiac tumours managed in the Children's Cardiac Centre that were not diagnosed prenatally. This is the Cardiac Centre for paediatric patients, where referrals from the entire country are sent. Therefore, according to our data, the overall prenatally diagnosed rate of primary cardiac tumours in our country in the analysed period was $43 \%$.

These children (not diagnosed prenatally) were referred for cardiology examination either by a neurologist in patients with tuberous sclerosis or by paediatricians in patients with a murmur.

The most important factor contributing to the percentage of prenatally diagnosed cases and the gestational age of the mother at the time of the diagnosis is the overall low number of cardiac defects that are diagnosed prenatally in our country. We believe that there is room for improvement in the prenatal diagnosis of foetal cardiac defects (comprising tumours) in Slovakia.

\section{Conclusion}

The primary foetal cardiac tumours are very rare malformations with variable prognoses. Once a primary cardiac tumour is diagnosed, further ultrasound and an echocardiographic monitoring of the foetus should be scheduled to modify the perinatal management according to the underlying foetal findings. Intracardiac flow obstruction, valves dysfunction and/or arrhythmias can lead to severe hemodynamic impairment, either prenatally or postnatally; these are the major factors contributing to the patient's final clinical outcome.

With the diagnosis of rhabdomyoma, the test for tuberous sclerosis should be performed since the tumour can be the first sign of the disease.

In cases of primary cardiac tumours, not only the prenatal diagnosis itself but also the correct stratification of high-risk patients is of great importance. It plays the key role in the optimal followup of the foetus, optimal delivery planning, as well as complex postnatal management, especially in cases where multidisciplinary approach is needed.

Our aim was to share our experiences with the diagnosis that may have a small incidence in the global population, but has a high impact on the individual and his family and the health care system alike.

\section{References}

1. Carrilho MC, Tonni G, Araujo Jr E. Fetal cardiac tumors: prenatal diagnosis and outcomes. Rev Braz Cir Cardiovasc 2015; 30: 6-7.

2. Lawley C, Popat H, Wong M, Badawi N, Ayer J. A dramatic response to Sirolimus therapy in a premature infant with massive cardiac rhabdomyoma. JACC: Case Reports 2019; 1 (3): 327-331.

3. Yuan SM. Fetal primary cardiac tumors during perinatal period. Pediatr Neonatol 2017; 58: 205-210.

4. Ebrahimi-Fakhari D, Mann LL, Poryo M et al. Incidence of tuberous sclerosis and age at first diagnosis: new data and emerging trends from a national, prospective surveillance study. Orphanet J Rare Dis 2018; 13 (117): $1-8$.

5. Frudit P, Vitturi BK, Navaroo FC, Rondelli I, Pozzan G. Multiple cardiac rhabdomyomas in tuberous sclerosis complex: case report and review of literature. Autops Case Rep (Internet) 2019; 9 (4): e2019125.https://doi. org/acr.2019.125.

6. Hoshal SG, Samuel BP, Schneider JR, Mammen I, Vettukattil JJ. Regression of massive cardiac rhabdomyoma on everolimus therapy. Pediatr Int 2016; 58: 397-399.

7. Aw F, Goyer I, Raboisson MJ, Boutin C, Major P, Dahnah N. Accelerated cardiac rhabdomyoma regression with everolimus in infants with tuberous sclerosis complex. Pediatr Cardiol 2017; 38 (2): 394-400.

8. Chen Y, Zeng Y, Zhu X et al. Significant difference between sirolimus and paclitaxel nanoparticles in anti proliferation effect in normoxia and hypoxia: The basis of better selection of atherosclerosis treatment. Bioact Mater 2021; 6 (3): 880-889.

9. Babu NM S, Behera D, Alex AG, Varghese L, George OK. Cardiac tumors in both twins-A case report af a rare occurrence. Ann Pediatr Cardiol 2020; 13 (3): 238-240.

10. Respondek-Liberska M. Fetal cardiac tumors. 44-45. In: Júnior EA, Bravo-Valenzuela M, Peixoto AB (Eds). Perinatal cardiology. Singapore, Bentham Science Publishers, 2020.

11. Bussani R, Castrichini M, Restivo $L$ et al. Cardiac tumors: diagnosis, prognosis and treatment. Curr Cardiol Rep 2020; 22 (169): 1-13.

12. Yuan SM, Yan SL, Wu N. Unusual aspects of cardiac myxoma. Anatol J Cardiol 2017; 17 (3): 241-247.

13. Yuan SM. Fetal cardiac tumors: clinical features, management and prognosis. J Perinat Med 2018; 46 (2): 115-121.

14. Malova J, Bohmar D, Luha J, Pastorakova A, Cierna Z, Braxatorisova T. Single umbilical artery and reproduction losses in Slovak population: relation to karyotype and fetal anomalies. Bratisl Med J 2018; 119 (6): 330-334.

15. Habanova H, Cunderlik A, Rusnak I. Fetal primary cardiac tumorscase study. Slov Gynek Pôrod 2019; 26: 170-172.

16. Ide T, Miyoshi T, Katsuragi S, Neki R, Kurosaki KI, Shiraishi I, Yoshimatsu J, Ikeda T. Prediction of postnatal arrhythmia in fetuses with cardiac rhabdomyoma. J Matern Fetal Neonatal Med 2019; 32 (15): 2463-2468.

17. Zhou QC, Fan P, Peng QH, Zhang M, Wang FU, Wang. Prenatal echocardiographic differential diagnosis of fetal cardiac tumors. Ultrasound Obstet Gynecol 2004; 23 (2): 165-171.

18. Zhen L, Yang YD, He Y et al. Prenatal genetic diagnosis of cardiac rhabdomyoma: A single-center experience. Eur J Obstet Gynecol Reprod Biol 2020; 249: 7-10.

Received November 9, 2020. Accepted December 9, 2020. 